\title{
Generalized quasiperiodic Rauzy tilings
}

\author{
Julien Vidal and Rémy Mosseri \\ Groupe de Physique des Solides, CNRS UMR 7588, \\ Universités Pierre et Marie Curie Paris 6 et Denis Diderot Paris 7, \\ 2 place Jussieu, 75251 Paris Cedex 05 France
}

\begin{abstract}
We present a geometrical description of new canonical $d$-dimensional codimension one quasiperiodic tilings based on generalized Fibonacci sequences. These tilings are made up of rhombi in $2 d$ and rhombohedra in $3 d$ as the usual Penrose and icosahedral tilings. Thanks to a natural indexing of the sites according to their local environment, we easily write down, for any approximant, the sites coordinates, the connectivity matrix and we compute the structure factor.
\end{abstract}

\section{INTRODUCTION}

Quasiperiodic tilings have been widely studied over the past decades, their main interest lying in their guessed relation with real quasicrystalline alloy 1 atomic structure. Indeed, most of the specific features and physical properties associated with quasiperiodic order (electronic structure, localized phason degree of freedom, nature and dynamics of dislocations, ...) are already pfesent in these simplified models. The two standard ways to generate these tilings are the cit and project methode.3 which relate them to selected pieces of higher dimensional lattices, and the grid algorithm 1 which insists on a dual picture of intersecting lines or planes. In both cases, one obtains structures made up of different types of tile which are segments in $1 d$, rhombi in $2 d$ and rhombohedra in $3 d$. The simplest (and most famous) exapples correspond to tilings with two differents tiles (the one-dimensional Fibonacci chain, the Penrose (pentagonal) 5 and Amman-Beenker (octogonal) two-dimensional tilings and the icosahedral $3 d$ tiling) although other quasiperiodic structures with more different types of tile have also been proposed. In $d \geq 2$, all these tilings display a complex local order with various coordination number.

Concerning the study of the physical properties (spectrum, eigenstates, conductivity,...), it has been proved more efficient to consider simpler systems such as direct products of periodic chainst 10 embedded in a quasiperiodic potential. The main advantage of these structures is that their characteristics (sub-ballistic transport, self-similar eigenstates) can be easily deduced from the $1 d$ case ; on the other hand, since all the sites have the same coordination number their topology is trivial. In $2 d$, Penrose-like tilings have nevertheless been studied revealing the same type of features11 13, ? 15 17 byt in $3 d$, the incidence of the topological quasiperiodicity on the electronic properties has been poorly investigated 18.19 essentially because of the geometrical complexity. In this context, it would be appreciable to have simple structures susceptible to provide a better framework for such studies.

The aim of this paper is to present new quasiperiodic tilings (of arbitrary dimension) following from a natural extension of the Fibonacci sequence, which allow for a coherent indexing of the sites while keeping some of the interesting self-similar properties of the Penrose-like tilings. Wegive the procedure to build any approximant of these topologically non trivial tilings using the conumbering scheme2021. In the quasiperiodic limit, these tilings are closely related to those initially considered by Rauzy 22 in two dimensions and will therefore be called generalized Rauzy tilings. In the first part, we recall the principle of the conumbering scheme that allows to generate all the sites of any codimension one structures by iterating the so-called generating vector $\mathbf{g}$. As an illustration, we apply this scheme to build the well-known Fibonacci chain for which it is possible to exactly determine the coordinates of $\mathbf{g}$. In the second part, we generalize these results in higher dimension and we describe, in details, the characteristics of the generalized Rauzy tilings in $2 d$ and $3 d$. The site coordinates are explicitely obtained in terms of the generalized Fibonacci numbers. In the third part, we give the connectivity matrix of these canonical tilings that is relevant for tight-binding electrons problems. The derivation of the structure factor is given in the fourth part. Finally, we propose, in the appendix, a dual point of view in which the $1 d$ quasiperiodic system presented have a high codimension although based on the same type of sequence.

\section{THE CONUMBERING SCHEME : APPLICATION TO THE FIBONACCI CHAIN}

In the cut and project algorithm commonly used to build quasiperiodic structures, one considers a $D$-dimensional hypercubic lattice and $d$-dimensional subspace that define the "physical" space. The $(D-d)$-dimensional subspace define the perpendicular space whose dimension is called the codimension. Thus, $d$-dimensional codimension one tilings are generated from a $(d+1)$-dimensional hypercubic lattice. The unidimensional character of the perpendicular space of these tilings provides a natural ordering of the sites which amounts to classify them according to their local 
environment : two sites with close coordinates in the perpendicular space have a similar neighbourhood. As shown below, each site of a codimension one tiling can actually be indexed by a unique coordonnate, its conumber, that is related to its position in the perpendicular space. For any approximant structure, this is achieved by using generating vector which, upon simple iteration, fully determines the coordinates of the sites inside the unit cell20.21. Since this method is valid for any approximant, the quasiperiodic structure can therefore be approached asymptotically.

To illustrate this mechanism, we shall focus on the $2 \rightarrow 1$ case. We consider a square lattice, and draw a line through the origin $O$, denoted $E^{\|}$(the parallel or physical space), of rational slope $\alpha=p / q$, with $p$ and $q$ mutually prime (see figure 1). The sites of the approximant structure are obtained by an orthogonal projection on $E^{\|}$of the square lattice sites contained inside the semi-open band generated by sliding the unit square along $E^{\|}$. Since $E^{\|}$has rational slope, it contains a set of regularly spaced sites of the square lattice which defines a unit cell with $n=|p|+|q|$ sites and a cell vector $\mathbf{A}^{\|}$. We define the so-called generating vector $\mathbf{g}$ as the vector of smallost norm 23 , joining the origin to the square lattice site closest to $E^{\|}$(but not belonging to $E^{\|}$). It can then be shown 2021 that the sites inside the band are obtained by successive translations of $\mathbf{g}$ ( $\left.\operatorname{modulo} \mathbf{A}^{\|}\right)$:

$$
\mathbf{r}^{j}=j \mathbf{g} \text { modulo } \mathbf{A}^{\|}, j \in[0, n-1] .
$$

This indexation by a unique coordinate (the conumber) provides a natural ordering with respect to the distance (before projection) from the parallel space, $i$. e. with respect to the local environment. Note that the origin has, by definition, the conumber 0 . For arbitrarysequences of approximants, the generator coordinates are given by a specific element of the Farey tree decomposition 20,21 but, for particular rational slopes, it is possible to explicitely write down the generating vector as we shall now see for the Fibonacci chain.

We denote by $\mathcal{B}_{2}=\left(\mathbf{e}_{1}, \mathbf{e}_{2}\right)$ the canonical orthonormal basis of the square lattice, and consider the vector $\mathbf{A}_{k}^{\perp}$ whose coordinates in $\mathcal{B}_{2}$ are given by $\left(F_{k}, F_{k-1}\right)$. The index $k$ refers to the approximant order and the Fibonacci sequence $\left(F_{l}\right)_{l \in \mathbf{Z}}$ is defined as follows :

$$
F_{l+1}=F_{l}+F_{l-1} \text { with } F_{0}=F_{1}=1
$$

Usually, this sequence is only defined for $l \geq 0$ but, here, we shall also consider negative values of $l$. Asreadily seen on the recursion relation (2) $\lim _{n \rightarrow \infty} F_{n+1} / F_{n}=\tau$ where the golden mean $\tau=(1+\sqrt{5}) / 2$ is the Pisot24 solution of the quadratic equation $x^{2}=x+1 . \quad \mathbf{A}_{k}^{\|}$is then defined as the vector of smallest norme 3 with integer coordinates perpendicular to $\mathbf{A}_{k}^{\perp}$. $\left(\mathbf{A}_{k}^{\|}, \mathbf{A}_{k}^{\perp}\right)$ forms a basis of the so-called trace lattice $\Lambda_{k}$. One also defines the band lattice $\Sigma_{k}$ generated by $\mathbf{A}_{k}^{\|}$and the vector $\mathbf{u}=(1,1)$ (see figure (1) for $k=4$ ). Let $L_{k}$ (resp. $S_{k}$ ) be the matrix transforming the canonical basis $\mathcal{B}_{2}$ into the $\Lambda_{k}$ (resp. $\Sigma_{k}$ ) basis. The number of sites of the square lattice contained in the $\Lambda_{k}$ (resp. $S_{k}$ ) unit cell thus reads $l_{k}=\left|\operatorname{det} L_{k}\right|\left(\right.$ resp. $\left.s_{k}=\left|\operatorname{det} S_{k}\right|\right)$. To determine the coordinate of the generating vector $\mathbf{g}_{k}$, we introduce the matrix $M$ :

$$
M=\left(\begin{array}{ll}
1 & 1 \\
1 & 0
\end{array}\right)
$$

that verifies the golden mean equation :

$$
M^{2}=M+1 \text {. }
$$




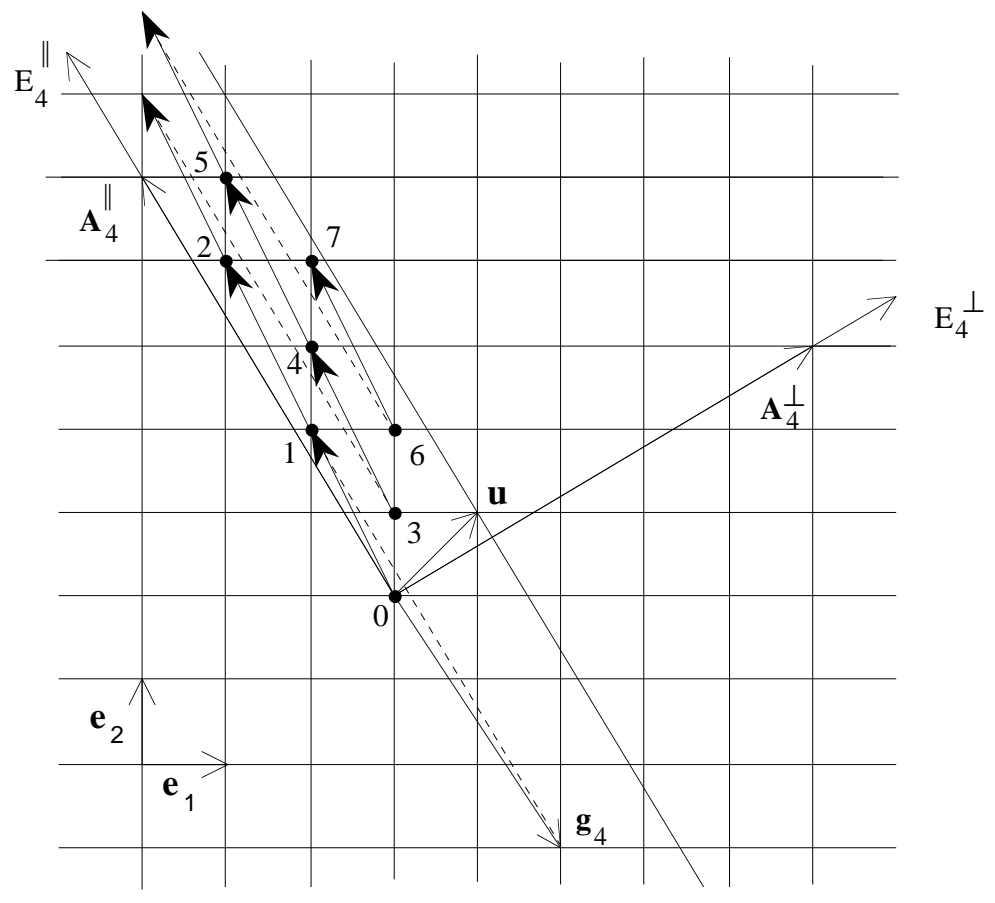

FIG. 1. Conumbering of the sites of the $4^{\text {th }}$ order approximant of the Fibonacci chain upon iteration of a generating vector. $\mathbf{A}_{4}^{\perp}=(5,3), \mathbf{A}_{4}^{\|}=(-3,5)$ and $\mathbf{g}_{4}=(2,-3)$.

The powers of $M$ can be expressed in terms of the Fibonacci numbers :

$$
M^{n}=\left(\begin{array}{ll}
F_{n} & F_{n-1} \\
F_{n-1} & F_{n-2}
\end{array}\right), \quad \forall n \in \mathbf{Z}
$$

Note that we also allow the index $n$ to be negative. Since the first column of $M^{k}$ represents the coordinates of $\mathbf{A}_{k}^{\perp}$, it comes immediately that the second line of $M^{-k}$ gives the coordinates of $\mathbf{A}_{k}^{\|}$in the basis $\mathcal{B}_{2}$ :

$$
\mathbf{A}_{k}^{\|}=\left(F_{-k-1}, F_{-k-2}\right) \text {. }
$$

The matrices $L_{k}$ and $S_{k}$ thus reads :

$$
L_{k}=\left(\begin{array}{ll}
F_{-k-1} & F_{k} \\
F_{-k-2} & F_{k-1}
\end{array}\right) \quad \text { and } \quad S_{k}=\left(\begin{array}{ll}
F_{-k-1} & 1 \\
F_{-k-2} & 1
\end{array}\right) .
$$

To determine the number of sites in the $\Sigma_{k}$ unit cell, one can either directly compute $s_{k}$ or one can remark that this number is also given by the scalar product of $\mathbf{u}$ and $\mathbf{A}_{k}^{\perp}$. One thus readily obtains :

$$
s_{k}=\mathbf{u} \cdot \mathbf{A}_{k}^{\perp}=F_{k}+F_{k-1}=F_{k+1} .
$$

To determine $\mathbf{g}_{k}$, one uses the fact that the quadrilateral generated by $\mathbf{A}_{k}^{\|}$and $\mathbf{g}_{k}$ should have a unit area since it cannot contain a lattice site (except on its boundary). Since $\mathbf{A}_{k}^{\|}$corresponds to the second line of $M^{-k}$ and since $\operatorname{det} M=-1$, the first line of $M^{-k}$ can be chosen to be the coordinates (in the basis $\mathcal{B}_{2}$ ) of the generating vector :

$$
\mathbf{g}_{k}=\left(F_{-k}, F_{-k-1}\right)
$$

The coordinates of the sites contained in the $\Sigma_{k}$ unit cell (sites of the approximant structure before projection), read in the basis $\mathcal{B}_{2}$ :

$$
\mathbf{r}_{k}^{j}=j \mathbf{g}_{k} \operatorname{modulo} \mathbf{A}_{k}^{\|}, j \in\left[0, s_{k}-1\right]
$$


The modulo operator allows to carry back all the sites in the same elementary cell of the band lattice. In the basis $\left(\mathbf{A}_{k}^{\|}, \mathbf{A}_{k}^{\perp}\right)$ of the trace lattice $\Lambda_{k}$, the coordinates finally write :

$$
\mathbf{r}_{k}^{j}=\operatorname{Frac}\left(j L_{k}^{-1} \mathbf{g}_{k}\right), j \in\left[0, s_{k}-1\right],
$$

where Frac (r) represents the fractional part of the $\mathbf{r}$ coordinates. The interest of introducing the trace lattice is that the coordinates of the sites after projection onto $E_{k}^{\|}$are readily given by the first component of $\mathbf{r}_{k}^{j}$ expressed in the $\left(\mathbf{A}_{k}^{\|}, \mathbf{A}_{k}^{\perp}\right)$ basis.

In the next section, we propose to generalize the above construction to codimension one structures in any dimension, such that the perpendicular direction is related to generalized Fibonacci numbers. These numbers are obtained from the Pisot solution of the polynomial equation $x^{D}=\sum_{j=0}^{D-1} x^{j}$, the $D=2$ case giving the golden mean.

\section{THE GENERALIZED RAUZY TILINGS}

The first geometrical censtruction based on the roots of this equation (for $D=3$ ) was proposed in 1982 by the mathematician G. Rauzy 22. Detailed analyses of these original tilings focusing on their self-similar properties and their fractal boundaries can be found in Refs.26 28. Nevertheless, the tilings that we describe thereafter are different although close to those initially studied. Indeed, the construction proposed by Rauzy does not rely on the standard cut and project algorithm since the sites of the cubic lattice chosen for the projection step are contained in a cylinder centered around the perpendicular direction and not in the band lattice. In addition, the construction of the approximant structures is completely new.

In order to show the simplicity of the generalization, we will use, in the following, the same notations for the $D \rightarrow D-1$ tilings as for the $2 \rightarrow 1$ tilings.

\section{A. The two-dimensional case}

As for the $2 \rightarrow 1$ case, we shall use the conumbering scheme to generate the $3 \rightarrow 2$ approximant structures. We endow the standard cubic lattice of a canonical orthonormal basis $\mathcal{B}_{3}=\left(\mathbf{e}_{1}, \mathbf{e}_{2}, \mathbf{e}_{3}\right)$ and, by analogy with the Fibonacci chain, we choose as perpendicular space the direction defined by vector $\mathbf{A}_{k}^{\perp}=\left(F_{k}, F_{k-1}, F_{k-2}\right)$ where the generalized Fibonacci sequence $\left(F_{l}\right)_{l \in \mathbf{Z}}$ is defined as follows :

$$
F_{l+1}=F_{l}+F_{l-1}+F_{l-2} \text { with } F_{-1}=0, F_{0}=F_{1}=1 .
$$

As previously, the ratio of two successive elements of this sequence converges toward an irrational limit :

$$
\lim _{n \rightarrow \infty} F_{n+1} / F_{n}=\alpha=\frac{4+(19+3 \sqrt{33})^{1 / 3}+(19+3 \sqrt{33})^{2 / 3}}{3(19+3 \sqrt{33})^{1 / 3}} \simeq 1.83929,
$$

where $\alpha$ is the Pisot root of the cubic equation $x^{3}=x^{2}+x+1$. We introduce the matrix $M$ :

$$
M=\left(\begin{array}{lll}
1 & 1 & 1 \\
1 & 0 & 0 \\
0 & 1 & 0
\end{array}\right)
$$

which satisfies $M^{3}=M^{2}+M+1$, and whose successive powers read :

$$
M^{n}=\left(\begin{array}{lll}
F_{n} & F_{n-1}+F_{n-2} & F_{n-1} \\
F_{n-1} & F_{n-2}+F_{n-3} & F_{n-2} \\
F_{n-2} & F_{n-3}+F_{n-4} & F_{n-3}
\end{array}\right) \quad \forall n \in \mathbf{Z}
$$

Below are shown the first (positive and negative) powers of $M$ which display a remarkable pattern on both parts of the identity matrix $M^{0}$. 


$\begin{array}{lrrrl} & 7 & 6 & 4 & \\ M^{4} & 4 & 3 & 2 & \\ & 2 & 2 & 1 & M^{3} \\ M^{2} & 1 & 1 & 1 & \\ & \mathbf{1} & \mathbf{0} & \mathbf{0} & M \\ M^{0} & \mathbf{0} & \mathbf{1} & \mathbf{0} & \\ & \mathbf{0} & \mathbf{0} & \mathbf{1} & M^{-1} \\ M^{-2} & 1 & -1 & -1 & \\ & -1 & 2 & 0 & M^{-3} \\ M^{-4} & 0 & -1 & 2 & \\ & 2 & -2 & -3 & \end{array}$

Note that the vertical sequences of numbers, in each column, obey relation (12) from bottom to top. To build the $k^{\text {th }}$ order approximant, one remarks that $\mathbf{A}_{k}^{\perp}$ corresponds to the first column of $M^{k}$ so that the two vectors $\mathbf{A}_{k}^{\|^{1}}$ and $\mathbf{A}_{k}^{\|^{2}}$ which generate the parallel space are directly obtained from the second and third line of $M^{-k}$ :

$$
\begin{aligned}
& \mathbf{A}_{k}^{\|^{1}}=\left(F_{-k-1}, F_{-k-2}+F_{-k-3}, F_{-k-2}\right) \\
& \mathbf{A}_{k}^{\|^{2}}=\left(F_{-k-2}, F_{-k-3}+F_{-k-4}, F_{-k-3}\right) .
\end{aligned}
$$

$\left(\mathbf{A}_{k}^{\|^{1}}, \mathbf{A}_{k}^{\|^{2}}, \mathbf{A}_{k}^{\perp}\right)$ defines a basis of the trace lattice $\Lambda_{k}$; the band lattice $\Sigma_{k}$ is generated by $\left(\mathbf{A}_{k}^{\|^{1}}, \mathbf{A}_{k}^{\|^{2}}\right)$ and the vector $\mathbf{u}=(1,1,1)$, which joins the origin to the extremity of the unit cube whose projection onto $\mathbf{A}_{k}^{\perp}$ has the highest positive magnitude. The number of sites in a $\Sigma_{k}$ unit cell is given by :

$$
s_{k}=\mathbf{u} . \mathbf{A}_{k}^{\perp}=F_{k}+F_{k-1}+F_{k-2}=F_{k+1} .
$$

The generating vector $\mathbf{g}_{k}$ is determined by the condition $: \operatorname{det}\left(\mathbf{A}_{k}^{\|^{1}}, \mathbf{A}_{k}^{\|^{2}}, \mathbf{g}_{k}\right)=1$. Since $\operatorname{det}\left(M^{-k}\right)=(\operatorname{det} M)^{-k}=1$, one can identify the coordinates of $\mathbf{g}_{k}$ with the first line of $M^{-k}$ :

$$
\mathbf{g}_{k}=\left(F_{-k}, F_{-k-1}+F_{-k-2}, F_{-k-1}\right) .
$$

and the coordinates (before projection) of the sites contained in a $\Sigma_{k}$ unit cell read in the basis $\mathcal{B}_{3}$ :

$$
\mathbf{r}_{k}^{j}=j \mathbf{g}_{k} \text { modulo }\left(\mathbf{A}_{k}^{\|^{1}}, \mathbf{A}_{k}^{\|^{2}}\right), j \in\left[0, s_{k}-1\right] .
$$

It is still interesting to express these coordinates in the trace lattice basis $\left(\mathbf{A}_{k}^{\|^{1}}, \mathbf{A}_{k}^{\|^{2}}, \mathbf{A}_{k}^{\perp}\right)$ :

$$
\mathbf{r}_{k}^{j}=\operatorname{Frac}\left(j L_{k}^{-1} \mathbf{g}_{k}\right), j \in\left[0, s_{k}-1\right]
$$

where :

$$
L_{k}=\left(\begin{array}{lll}
F_{-k-1} & F_{-k-2} & F_{k} \\
F_{-k-2}+F_{-k-3} & F_{-k-3}+F_{-k-4} & F_{k-1} \\
F_{-k-2} & F_{-k-3} & F_{k-2}
\end{array}\right)
$$

After projection onto $E_{k}^{\|}$, the $j^{\text {th }}$ site coordinates, in the basis $\left(\mathbf{A}_{k}^{\|^{1}}, \mathbf{A}_{k}^{\|^{2}}\right)$ are given by the two first components of $\mathbf{r}_{k}^{j}$. Figure 2 displays an elementary cell of the $10^{t h}$ order approximant. 


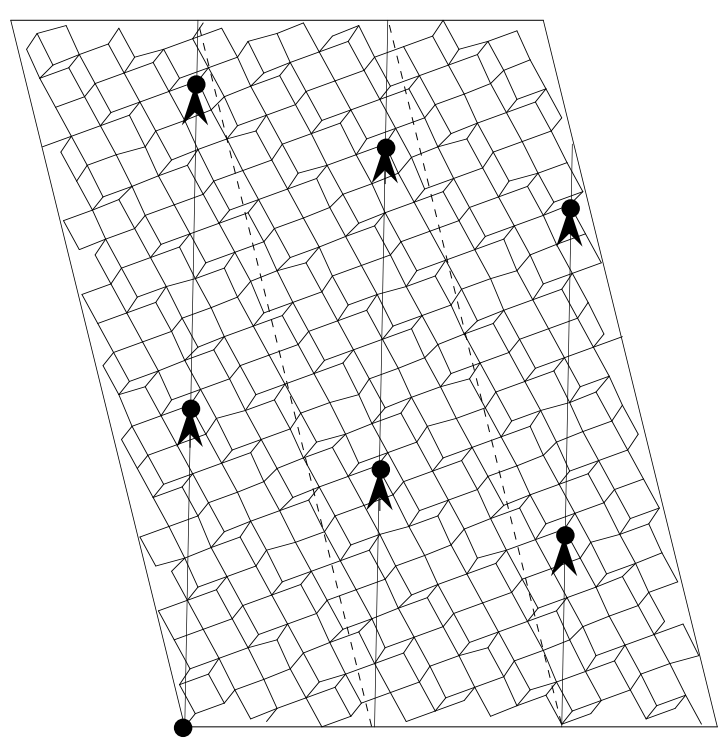

FIG. 2. Unit cell of the $10^{\text {th }}$ order approximant (504 sites). The first iterations of the generator are shown.

In the initial cubic lattice, each site is six-fold coordinated so that, after projection, the coordination number $z$ is such that $3 \leq z \leq 6$. However, as discussed below, the tilings generated here only have sites with $3 \leq z \leq 5$.

\section{B. The higher dimensional case}

The simplicity of the above construction results directly from the particular properties of the generalized Fibonacci numbers. It can therefore easily extended to codimension one tilings of any higher dimension. Thereafter, we briefly describe the three-dimensional case and the $d$-dimensional case.

The construction in the $4 \rightarrow 3$ case is based on the Pisot root of the equation :

$$
x^{4}=x^{3}+x^{2}+x+1
$$

whose approximants define the perpendicular space direction in the $4 D$ space.

The associated generalized Fibonacci sequence $\left(F_{l}\right)_{l \in \mathbf{Z}}$ is defined by :

$$
F_{l+1}=F_{l}+F_{l-1}+F_{l-2}+F_{l-3} \text { with } F_{-1}=F_{-2}=0, F_{0}=F_{1}=1,
$$

and the $M$ matrix satisfying (24) reads :

$$
M=\left(\begin{array}{llll}
1 & 1 & 1 & 1 \\
1 & 0 & 0 & 0 \\
0 & 1 & 0 & 0 \\
0 & 0 & 1 & 0
\end{array}\right)
$$

so that :

$$
M^{n}=\left(\begin{array}{llll}
F_{n} & F_{n-1}+F_{n-2}+F_{n-3} & F_{n-1}+F_{n-2} & F_{n-1} \\
F_{n-1} & F_{n-2}+F_{n-3}+F_{n-4} & F_{n-2}+F_{n-3} & F_{n-2} \\
F_{n-2} & F_{n-3}+F_{n-4}+F_{n-5} & F_{n-3}+F_{n-4} & F_{n-3} \\
F_{n-3} & F_{n-4}+F_{n-5}+F_{n-6} & F_{n-4}+F_{n-5} & F_{n-4}
\end{array}\right) \quad \forall n \in \mathbf{Z}
$$

The $k^{t h}$ order approximant is obtained by choosing the vector $\mathbf{A}_{k}^{\perp}=\left(F_{k}, F_{k-1}, F_{k-2}, F_{k-3}\right)$ whose coordinates are given by the first column of $M^{k}$. The three parallel space vectors reads : 


$$
\begin{aligned}
& \mathbf{A}_{k}^{\|^{1}}=\left(F_{-k-1}, F_{-k-2}+F_{-k-3}+F_{-k-4}, F_{-k-2}+F_{-k-3}, F_{-k-2}\right) \\
& \mathbf{A}_{k}^{\|^{2}}=\left(F_{-k-2}, F_{-k-3}+F_{-k-4}+F_{-k-5}, F_{-k-3}+F_{-k-4}, F_{-k-3}\right) \\
& \mathbf{A}_{k}^{\|^{3}}=\left(F_{-k-3}, F_{-k-4}+F_{-k-5}+F_{-k-6}, F_{-k-4}+F_{-k-5}, F_{-k-4}\right),
\end{aligned}
$$

that are respectively the second, third and fourth line of $M^{-k}$. The band lattice $\Sigma_{k}$ is generated by $\left(\mathbf{A}_{k}^{\|^{1}}, \mathbf{A}_{k}^{\|^{2}}, \mathbf{A}_{k}^{\|^{3}}\right)$ and the vector $\mathbf{u}=(1,1,1,1)$. The number of sites in the $\Sigma_{k}$ unit cell (and therefore in the approximant unit cell) is given by :

$$
s_{k}=\mathbf{u} \cdot \mathbf{A}_{k}^{\perp}=F_{k}+F_{k-1}+F_{k-2}+F_{k-3}=F_{k+1},
$$

and the coordinates of the generating vector are given by the first line of $M^{-k}$ :

$$
\mathbf{g}_{k}=\left(F_{-k}, F_{-k-1}+F_{-k-2}+F_{-k-3}, F_{-k-1}+F_{-k-2}, F_{-k-1}\right) \text {, }
$$

The sites coordinates have an expression similar to Eq.(22) where $L_{k}$ is, as before, built from the coordinates of $\mathbf{A}_{k}^{\|^{1}}, \mathbf{A}_{k}^{\|^{2}}, \mathbf{A}_{k}^{\|^{3}}$ and $\mathbf{A}_{k}^{\perp}$.

The generalization in any dimension $D$ is straightforward considering the Pisot root of the equation :

$$
x^{D}=\sum_{j=0}^{D-1} x^{j},
$$

The remaining of the construction follows mechanically.

Let us end this section by remarking that nothing prevents, in any dimension, to map the whole structure along a direction such that all tiles (rhombus in $2 d$, rhombohedra in $3 d$ ) are identical up to rotations. Indeed, once the sites have been generated with a given choice of the perpendicular space, this can be achieved by projecting perpendicularly to the direction of the vector $\mathbf{u}$ whose each coordinate equals 1 . In that case, all the sites are mapped onto the full set of simple lattice vertices (triangular in $2 d$ and centered cubic in $3 d$ ) with missing edges. We are then left with a quasiperiodic decoration of a periodic structure, which has the same connectivity but a different geometry as the above discussed tilings. Such deformations might be useful in some context, like for instance when one aims to build row-by-row or plane-by-plane transfer matrices, in which case the preexisting reticular stratification of the lattice can be very helpful.

\section{CONNECTIVITY MATRIX OF CODIMENSION ONE TILINGS}

In this section we write down the so-called connectivity or adjacency matrix $K$ of codimension one tilings. This matrix is defined by the following rule : $K_{i j}=1$ if sites $i$ and $j$ are nearest neighbours ( $i$. e. connected by an edge) and zero otherwise. Such a matrix is of special interest for the study of the electronic or phononic excitations in tight-binding like approaches. In this case, the hamiltonian is, indeed, simply proportional to K. Being able to easily obtain such a matrix may therefore be highly valuable.

The idea consists in labelling the sites with respect to their conumber. As discussed in section II, this indexation classifies the sites according to their coordinate in the perpendicular space direction, or equivalently, in terms of their local environment. This implies that the conumber difference between two nearest neighbour sites along a given direction is a constant modulo the number of sites in the unit cell. This difference is readily obtained from the projection onto the perpendicular space of each edges meeting at any site of the initial hypercubic lattice. It is therefore sufficient to determine, for a given site, the conumber of its nearest neighbours, to write down the full connectivity matrix as a band (Tœplitz-like) matrix.

We shall first illustrate this method with a Fibonacci chain approximant, defined by the perpendicular space vector $\mathbf{A}_{k}^{\perp}=\left(F_{k}, F_{k-1}\right)$ and determine the conumber of the nearest neighbours of the origin. In the canonical basis $\mathcal{B}_{2}$ of the square lattice, these sites have the following coordinates $(0, \pm 1)$ and $( \pm 1,0)$. Their projection onto the perpendicular space is given (up to a sign), in the trace lattice $\Lambda_{k}$ basis, by $\mathbf{A}_{k}^{\perp} / l_{k}$. In addition, and by construction, one has $\mathbf{A}_{k}^{\perp} \cdot \mathbf{g}_{k}=1 / l_{k}$ in the $\Lambda_{k}$ basis. One therefore deduces that the conumber of the sites which are nearest neighbours of the origin (once carried back in a unique unit cell) reads $F_{k}$ et $F_{k-1}$. 
As an example, we give the connectivity matrix $K$ of the $4^{\text {th }}$ order approximant of the Fibonacci chain displayed in figure (1) with periodic boundary conditions:

$$
K=\left(\begin{array}{llllllll}
0 & 0 & 0 & 1 & 0 & 1 & 0 & 0 \\
0 & 0 & 0 & 0 & 1 & 0 & 1 & 0 \\
0 & 0 & 0 & 0 & 0 & 1 & 0 & 1 \\
1 & 0 & 0 & 0 & 0 & 0 & 1 & 0 \\
0 & 1 & 0 & 0 & 0 & 0 & 0 & 1 \\
1 & 0 & 1 & 0 & 0 & 0 & 0 & 0 \\
0 & 1 & 0 & 1 & 0 & 0 & 0 & 0 \\
0 & 0 & 1 & 0 & 1 & 0 & 0 & 0
\end{array}\right)
$$

There are indeed $F_{5}=8$ sites in the elementary cell and the conumber of the origin neighbours are $F_{4}=5$ and $F_{3}=3$ (see fig. 11).

The above discussion applies to the generalized Rauzy tilings : their connectivity matrix remains a band matrix once the sites have been ordered according to their conumber. The conumbers of the origin's nearest neighbours are still given by the coordinates of $\mathbf{A}_{k}^{\perp}$ which are expressed in terms of the generalized Fibonacci numbers. We display below the connectivity matrix of the $4^{\text {th }}$ order approximant of the two-dimensional generalized Rauzy tiling, with periodic boundary conditions :

$$
K=\left(\begin{array}{lllllllllllll}
0 & 0 & 1 & 0 & 1 & 0 & 0 & 1 & 0 & 0 & 0 & 0 & 0 \\
0 & 0 & 0 & 1 & 0 & 1 & 0 & 0 & 1 & 0 & 0 & 0 & 0 \\
1 & 0 & 0 & 0 & 1 & 0 & 1 & 0 & 0 & 1 & 0 & 0 & 0 \\
0 & 1 & 0 & 0 & 0 & 1 & 0 & 1 & 0 & 0 & 1 & 0 & 0 \\
1 & 0 & 1 & 0 & 0 & 0 & 1 & 0 & 1 & 0 & 0 & 1 & 0 \\
0 & 1 & 0 & 1 & 0 & 0 & 0 & 1 & 0 & 1 & 0 & 0 & 1 \\
0 & 0 & 1 & 0 & 1 & 0 & 0 & 0 & 1 & 0 & 1 & 0 & 0 \\
1 & 0 & 0 & 1 & 0 & 1 & 0 & 0 & 0 & 1 & 0 & 1 & 0 \\
0 & 1 & 0 & 0 & 1 & 0 & 1 & 0 & 0 & 0 & 1 & 0 & 1 \\
0 & 0 & 1 & 0 & 0 & 1 & 0 & 1 & 0 & 0 & 0 & 1 & 0 \\
0 & 0 & 0 & 1 & 0 & 0 & 1 & 0 & 1 & 0 & 0 & 0 & 1 \\
0 & 0 & 0 & 0 & 1 & 0 & 0 & 1 & 0 & 1 & 0 & 0 & 0 \\
0 & 0 & 0 & 0 & 0 & 1 & 0 & 0 & 1 & 0 & 1 & 0 & 0
\end{array}\right)
$$

One has $F_{5}=13$ sites in the unit cell, and the conumber of the origin's nearest neighbours are $F_{4}=7, F_{3}=4$, and $F_{2}=2$. One can therefore appreciate the advantage of this family of tilings that provides topologically non trivial structures which are nevertheless very easy to construct and to encode. Especially in $3 d$, this is undoubtlessly one of the simplest type of connectivity matrix that one could expect for quasiperiodic structure approximant of any order.

The last point we would like to discuss concerns the proportion of the different types of site and their coordination number. Clearly, the coordination number of site $j$ is the number of 1 in the $j^{\text {th }}$ line of the connectivity matrix $K$. Hence, in the above example one finds four 3-fold coordinated sites, five 4-fold coordinated sites, and four 5-fold coordinated sites but no 6-fold coordinated sites. It is easy to show that the condition to have sites of maximal coordination number $z=2 D$ in a generalized Rauzy tiling of type $D \rightarrow(D-1)$ reads :

$$
F_{k+1}-2 F_{k}>0 \Leftrightarrow \frac{F_{k+1}}{F_{k}}>2 \text {. }
$$

But looking to the definition of the generalized Fibonacci sequence $\left(F_{l}\right)_{l \in \mathbf{Z}}$ in the $D$-dimensional case :

$$
F_{n+1}=\sum_{j=0}^{D-1} F_{n-j} \text { with } F_{j}=0 \text { for } j \in[2-D,-1] \text { and } F_{0}=F_{1}=1 \text {, }
$$

one can easily prove, by recursion, that the condition (36) is neyer fulfilled. This is in contrast with the two-dimensional tilings originally introduced by Rauzy and discussed in Ref.26 that displayed 6-fold coordinated sites. 


\section{STRUCTURE FACTOR}

The simple form taken by the codimension 1 tiling sites coordinates is very helpful to compute the structure factor (which amounts here to the Fourier transform of the site-distribution). Indeed, the existence of a generating vector allows to write the structure factor as a geometric serie21. Denoting by $n$ the number of sites in a unit cell and assuming that the sites (atoms) have the same form factor, the structure factor $S$ reads :

$$
S(\mathbf{q})=\frac{1}{n} \sum_{j=0}^{n-1} e^{i \mathbf{q} \cdot \mathbf{r}^{j}}
$$

where $\mathbf{q}$ is a reciprocal space vector of the approximant structure and where $\mathbf{r}^{j}$ represents the vector associated to the site $j$ (after projection). In the codimension one case, we have seen that the coordinates were simply expressed in terms of the generator $\mathbf{g}$ :

$$
\mathbf{r}^{j}=j \mathbf{g}, \quad j \in[0, n-1] .
$$

This expression slighly differs from (21) since here, $\mathbf{r}^{j}$ and $\mathbf{g}$ denotes the vectors after projection. In addition, the sites are not carried back in the unit cell through the modulo operation, but since one only considers reciprocal space vector, this does not affect the result. Thus, the structure factor $S$ simply writes :

$$
S(\mathbf{q})=\frac{1}{n} \sum_{j=0}^{n-1} e^{i j \mathbf{q} \cdot \mathbf{g}} .
$$

The peak intensities (think of a diffraction experiment) are proportional to :

$$
|S(\mathbf{q})|=\frac{1}{n}\left|\frac{\sin (n \mathbf{q} \cdot \mathbf{g} / 2)}{\sin (\mathbf{q} \cdot \mathbf{g} / 2)}\right| .
$$

In the quasiperiodic limit, the reciprocal space unit cell shrinks toward zero, and the Fourier spectrum becomes dense. However, going from an approximant to the next one mostly amounts to add new peaks of smaller and smaller intensities while letting almost unchanged the previous peaks. This is why any threshold function filtering out the peaks below a certain (arbitrary) value gives rise to a point-like diffraction pattern (see figure (3) for an approximant of a two-dimensional generalized Rauzy tiling).

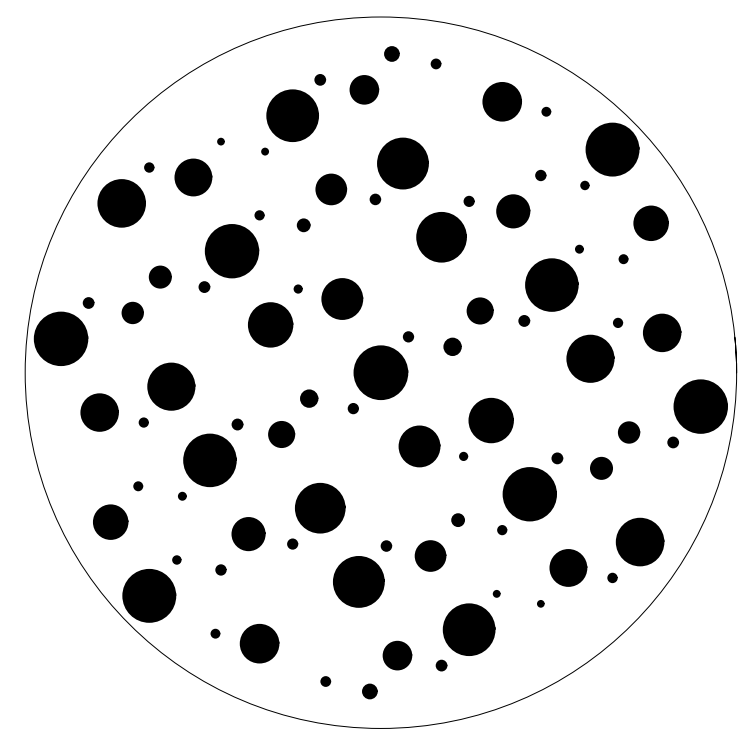

\footnotetext{
${ }^{1}$ Since approximant structures are periodic, only those vectors contribute to the structure factor.
} 
FIG. 3. Structure factor for the $10^{\text {th }}$ order approximant (504 sites per unit cell). Only intensities greater than an arbitrary threshold are shown. The radius of the circles is proportional to $|S|$.

Note that the Fourier spectrum does not display a particular point-like rotational symmetry (except the trivial parity $\mathbf{q} \leftrightarrow \mathbf{- q}$ ), in constrast with the more commonly studied octagonal and Penrose quasiperiodic tilings which have higher codimension ( 2 and 3 respectively).

\section{CONCLUSION}

The generalized Rauzy tilings form a canonical set of codimension one quasiperiodic structures. Their construction is based on the generalized Fibonacci sequence, whose properties allow for a rather simple method to get the sites coordinates as well as their coordination number. We have shown how to get closed formulas for these quantities valid for any approximant structures, and which therefore can be carried up to the quasiperiodic limit. The unidimensional nature of the perpendicular space allows one to write down the connectivity matrix in terms of Tœplitz band-like matrix, the position of the non vanishing band being directly determined from the generalized Fibonacci number

These properties should rank this family of tilings among the most interesting to be studied. As shown recently 2930 , they are, to many respect, much simpler than the celebrated Penrose-like tilings, while displaying the same kind of physical properties.

We would like to thank P. Arnoux, N. Destainville and L. Vuillon for very fruitful discussions about Rauzy original works.

\section{APPENDIX A: ONE-DIMENSIONAL GENERALIZED FIBONACCI CHAINS OF HIGH CODIMENSION}

Up to now, we have described $d$-dimensional codimension one structures with a perpendicular space built from the generalized Fibonacci series. But nothing prevents to build, along the same line, one-dimensional structures made up of $(d-1)$ different edges, by switching the respecting role of the perpendicular and parallel space. Let us briefly describe the obtained sequences and start by discussing the $3 \rightarrow 1$ case.

We write down the 3 -letter substitution which is given by the matrix $M$,

$$
A \rightarrow A B, B \rightarrow A C, C \rightarrow A
$$

which generates (starting from $A$ as an example), the following set of sequences $S_{l}$ :

$$
S_{1}=A, S_{2}=A B, S_{3}=A B A C, S_{4}=A B A C A B A, S_{5}=A B A C A B A A B A C A B, \ldots
$$

Note that the length of the $l^{\text {th }}$ sequence is given by the generalized Fibonacci numbers $\mathrm{F}_{l}$. The sequences follow a concatenation rule $S_{l+1}=S_{l} S_{l-1} S_{l-2}$ and the number of occurences of $A$ (resp. $B, C$ ) in the sequence $S_{l}$ is $F_{l-1}$ (resp. $F_{l-2}, F_{l-3}$ ). Note that a related sequence has actually been considered in a previous work 31 but with the reverse concatenation rule : $S_{l+1}=S_{l-2} S_{l-1} S_{l}$.

The generalization for higher codimension is straightforward.

${ }^{1}$ D. Shechtman, I. Blech, D. Gratias, and J. W. Cahn, Phys. Rev. Lett. 53, 1951 (1984).

${ }^{2}$ M. Duneau and A. Katz, Phys. Rev. Lett. 54, 2688 (1985).

${ }^{3}$ P. A. Kalugin, A. Y. Kitaev, and L. S. Levitov, J. Phys. (Paris) Lett. 46, L601 (1985).

${ }^{4}$ N. G. de Bruijn, Proc. Konink. Ned. Akad. Wetensch. A 43, C3 (1968). 
${ }^{5}$ R. Penrose, Bull. Inst. Math. Appl. 10, 266 (1974).

${ }^{6}$ F. P. M. Beenker, Univ. of Technology, Eindhoven T. H., Report WSK 1982. This tiling was also anticipated by Amman (unpublished).

7 J. X. Zhong and R. Mosseri, J. Phys. C 7, 8383 (1995).

${ }^{8}$ J. A. Ashraff, J. M. Luck, and R. B. Stinchcomb, Phys. Rev. B 41, 4314 (1990).

${ }^{9}$ K. Ueda and H. Tsunetsugu, Phys. Rev. Lett. 58, 1272 (1987).

${ }^{10}$ C. Sire, in Lectures on Quasicrystals, edited by F. Hippert and D. Gratias (Editions de Physique, Les Ulis France, 1994).

${ }^{11}$ M. Kohmoto and B. Sutherland, Phys. Rev. Lett. 56, 2740 (1986).

${ }^{12}$ H. Tsunetsugu and K. Ueda, Phys. Rev. B 43, 8892 (1991).

${ }^{13}$ T. Rieth, U. Grimm, and M. Schreiber, in Proceedings of the 6th International Conference on Quasicrystals, edited by S. Takeuchi and T. Fujiwara (World Scientific, Singapore, 1998), p. 639.

${ }^{14}$ P. Repetowicz, U. Grimm, and M. Schreiber, Phys. Rev. B 58, 13482 (1998).

${ }^{15}$ B. Passaro, C. Sire, and V. G. Benza, Phys. Rev. B 46, 13751 (1992).

${ }^{16}$ C. Sire and J. Bellissard, Europhys. Lett. 11, 439 (1990).

17 J. X. Zhong and R. Mosseri, J. Phys. (Paris) 4, 1513 (1994).

${ }^{18}$ H. Schwabe, G. Kasner, and H. Böttger, Phys. Rev. B 56, 8026 (1997).

${ }^{19}$ G. Kasner, H. Schwabe and H. Böttger, Phys. Rev. B 51, 10454 (1995).

${ }^{20}$ R. Mosseri, in Proceedings of the 3rd International Meeting on Quasicrystals, edited by J. Yacamán et al. (World Scientific, Singapore, 1990), p. 129.

${ }^{21}$ R. Mosseri, in Proceedings in Physics Vol. 32, Universalities in Condensed Matter, edited by R. Jullien et al. (Springer-Verlag, Berlin Heidelberg, 1988), p. 9.

${ }^{22}$ G. Rauzy, Bull. Soc. Math. France 110, 147 (1982).

23 The vectors $\mathbf{g}$ and $\mathbf{A}^{\perp}$ are defined up to a sign but each choice leads to the same structure. Here, by convention, we choose g such that $\mathbf{g} . \mathbf{A}^{\perp}>0$.

${ }^{24} \mathrm{~A}$ number, is said to be a Pisot number if it is a root of a polynomial with integer coefficients such that its modulus is bigger than one and all other roots moduli are smaller than one.

${ }^{25}$ M. Duneau, R. Mosseri, and C. Oguey, J. Phys. A 22, 4549 (1989).

${ }^{26}$ S. Hito and M. Kimura, Japan J. Indust. Appl. Math. 8, 461 (1991).

${ }^{27}$ A. Messaoudi, 1987, thèse de doctorat, Université de la Méditérranée, Aix-Marseille II.

${ }^{28}$ P. Arnoux and S. Ito, 1998, Pisot substitutions and Rauzy fractal, prépublication 98-18 de l'IML.

${ }^{29}$ J. Vidal and R. Mosseri, 1999, Proceedings of the 7th International Conference on Quasicrystals, to be published.

${ }^{30} \mathrm{~F}$. Triozon et al., in preparation.

${ }^{31}$ M.K. Ali and G. Gumbs, Phys. Rev. B 38, 7091 (1988). 